\title{
The risk factors for developing exposure keratopathy in ICU patients
}

\author{
O Kousha ${ }^{1}$, Z Kousha ${ }^{2 *}$, J Paddle ${ }^{1}$ \\ From ESICM LIVES 2015 \\ Berlin, Germany. 3-7 October 2015
}

\section{Introduction}

Intensive care unit (ICU) patients are at increased risk of developing exposure keratopathy (EK) due to intubation, sedation, paralysis and metabolic disturbance. The factors lead to reduced venous return from the eyes, impairment of the blink reflex, loss of the tone of the orbicularis oculi muscles and dysfunction of the corneal healing. EK can lead to short- and long-term visual impairment.

\section{Objectives}

To ascertain the risk factors for the development of EK in the whole ICU population.

\section{Methods}

A prospective cohort study of all patients admitted consecutively over a four months period, from 24/11/2014 to $31 / 03 / 2015$. A total of 257 patients were included and 17 were excluded ( 2 patients refused consent, 4 patients were too agitated, 3 patients had pre-existing ocular surface disease and 8 patients were below the age of 16). Every patient was assessed everyday using a standardised pro forma with a portable slit lamp. A total of 2712 eye assessments were carried out.

\section{Results}

The incidence of EK in mechanically ventilated patients was $54.3 \%$ compared to $5.1 \%$ in patients receiving noninvasive ventilation or no ventilatory support $(p<$ 0.001). Relative risk was 10.6 (95\% confidence Interval (CI) 5.5 to 20.7). A logistic regression model was constructed to calculate adjusted odds ratios (OR) for various factors (Table 1). Mechanical ventilation and lagophthalmos were identified as the main risk factor

${ }^{2}$ University College London, Faculty of Medical Sciences, London, United Kingdom

Full list of author information is available at the end of the article
Table 1 Adjusted OR of developing EK for various factors

\begin{tabular}{|c|c|c|}
\hline Risk Factors & OR $(95 \% \mathrm{Cl})$ & $p$-value \\
\hline Male Gender & $1.1(0.5-2.7)$ & 0.733 \\
\hline $\mathrm{Age}^{\mathrm{a}}$ & $1.0(1.0-1.1)$ & 0.191 \\
\hline APACHE II score ${ }^{b}$ & $1.1(1.0-1.1)$ & 0.104 \\
\hline Mechanical ventilation & $6.8(3.2-8.0)$ & 0.028 \\
\hline Sedation & $1.2(0.3-7.7)$ & 0.068 \\
\hline Lagophthalmos & $32.5(15.3-45.1)$ & $<0.001$ \\
\hline
\end{tabular}

for developing EK with lagophthalmos having the largest effect. Among mechanically ventilated patients who received less than 3 times daily eye care, the incidence of EK was $64.5 \%$ compared to $11.5 \%$ in the patients receiving eye care 3 times a day or more $(p<0.001)$. Relative risk was 5.6 (95\% CI 3.8 to 7.7 ).

\section{Conclusions}

EK is a common but preventable condition in ICU patients with the major risks being mechanical ventilation and lagophthalmos. However, prevention and treatment strategies can be developed to identify the patients at risk, prevent the development of EK and, if EK develops, to treat EK.

\section{Authors' details}

${ }^{1}$ Royal Cornwall Hospital NHS Trust, Truro, United Kingdom. ${ }^{2}$ University College London, Faculty of Medical Sciences, London, United Kingdom.

Published: 1 October 2015

doi:10.1186/2197-425X-3-S1-A731

Cite this article as: Kousha et al:: The risk factors for developing

exposure keratopathy in ICU patients. Intensive Care Medicine Experimental 2015 3(Suppl 1):A731. (c) 2015 Kousha et al.; This is an Open Access article distributed under the terms of the Creative Commons Attribution License (http:// creativecommons.org/licenses/by/4.0), which permits unrestricted use, distribution, and reproduction in any medium, provided the original work is properly cited. 